\title{
Towards developing collaborative experience based factory model for software development process in cloud computing environment
}

\begin{abstract}
Knowledge and experience are important assets for software organizations. In todayô global software development trends, development teams are no longer located in single premise; they are spreading across national and geographic boundaries. As a software project progresses, more and more activities are involved which results with the accumulation of knowledge and experiences. Maintaining and reusing of past experiences are vital; and it is even more crucial for distributed teams. In order to sustain in todayô competitive advantages, organizations should prepare a well collaborative solution for managing software development knowledge and experiences to maximize sharing and future reuse. Numerous attempts have been invested by researchers to overcome the issues on knowledge management in software development; however, the emphasis on the actual experiences collected throughout the development phases is limited. Furthermore, there are not many solutions offering comprehensive collaborative solution for managing software development experiences. In this paper, we propose a model for managing software development experiences including its tacit and explicit knowledge based on experience factory approach. The model is adapted for cloud computing environment with the goal to provide efficient and effective collaborative solution for knowledge access, sharing and reuse by capitalizing the cloudôs resources and infrastructure. A systematic literature review has been conducted to investigate the current issues of knowledge management in software development and to analyze available approaches and solutions. The findings are quantitatively and qualitatively evaluated to support the model formulation.
\end{abstract}

Keyword: Cloud computing; Collaborative; Experience based factory; Knowledge management; Software development process 